\title{
AN EXTENSION OF THE METHOD OF AVERAGING*
}

\author{
BY
}

P. R. SETHNA

Brown University

I. Introduction. Consider the real system of differential equations

$$
\dot{x}=\epsilon F(t, x, \epsilon)
$$

where $x$ is an $n$ vector $0<\epsilon \leqq \epsilon_{0}$ and $F$ is a continuous function from

$B\left(K, \epsilon_{0}\right)=\{(t, x, \epsilon): \mathrm{t}$ and $\epsilon$ scalars, $x$ an $n$ vector,

$$
\left.-\infty<t<\infty, 0 \leqq|x| \leqq K, 0<\epsilon \leqq \epsilon_{0}\right\}
$$

into $R^{n}$. Consider also the averaged system of equations

$$
\dot{\xi}=\epsilon F_{0}(\xi)
$$

where

$$
F_{0}(\xi)=\lim _{T \rightarrow \infty} \frac{1}{T} \int_{0}^{T} F(t, \xi, \epsilon) d t .
$$

The method of averaging [1] $]^{1},[2]$ is concerned with establishing relationships between solutions (or integral manifolds) of (1.1) and solutions of (1.2). In general, it shows that the existence and stability properties of certain solutions of (1.2) determine the existence and stability of the corresponding solutions of (1.1) and that solutions of (1.1) approach in some sense the solutions of (1.2) as $\epsilon \rightarrow 0$. In its standard form the results of the method fall into two categories. They are, respectively, results valid for finite and infinite time intervals. For the finite time results one merely requires the existence of (1.3), and the solutions of (1.1) and (1.2), starting with the same initial conditions, are shown to stay close to each other at each $t$, in a time interval proportional to $\epsilon^{-1}$, for $\epsilon$ sufficiently small. For the results valid for infinite time, $F$ is assumed to be almost periodic in $t$ uniformly in $x$ for each fixed $\epsilon, 0<\epsilon \leqq \epsilon_{0}$, and it is shown that almost periodic solutions of (1.1) stay close to certain constant solutions of (1.2) for all time, for $\epsilon$ sufficiently small. The results as established for equations of the type (1.1) have been applied very successfully to numerous problems of physical importance.

There are, however, a large class of physical problems which generate differential equations more general than (1.1) in which the function $F$ depends not only on the time $t$ but also on a slow time $\epsilon$. Specifically, the system equations take the form

$$
\dot{x}=\epsilon F(t, \epsilon t, x, \epsilon)
$$

where $x$ and $F$ are $n$ vectors, $0<\epsilon \leqq \epsilon_{0}$ and $F$ is a continuous function of its arguments for all $t$ and $0 \leqq|x| \leqq K$. One can again construct an averaged system corresponding to

*Received July 28, 1966.

'Numbers in square brackets refer to references at the end of the paper. 
(1.4) by averaging over $t$ and regarding $\epsilon t$ as constant during averaging. With appropriate conditions on the function $F$, results analogous to the finite time results for the standard form of the method of averaging can be obtained. See, for instance, Mitropolsky [3] and also Volosov [4] who treats systems even more general than (1.4). Although these results are useful in many technical applications there are other applications where results valid for finite time intervals are inadequate.

Consider for example a physical system which can be described by the system of equations

$$
\frac{d x}{d t}=G(\nu t, \omega t, x)
$$

where $x$ is an $n$ vector and $G$ is a continuous function of its arguments into $R^{n}$. In equation (1.5) $t, \omega$ and $\nu$ are real, $x$ an $n$ vector, $-\infty<t<\infty, 0 \leqq|x| \leqq K$ and $\omega>0$, $\nu>0$. Let $G$ be periodic in $\omega t$ and $\nu t$ with period $2 \pi$ and suppose $\nu=\epsilon^{-1}$ where $\epsilon$ is very small and positive and let $\omega$ be of order zero in $\epsilon$. Now let $\tau=\nu t=\epsilon^{-1} t$ be the "fast time", then (1.5) takes the form

$$
\frac{d x}{d \tau}=\epsilon G(\tau, \omega \epsilon \tau, x)
$$

Now (1.6) is of the form (1.4), but results valid for $\tau \varepsilon\left[0, L \epsilon^{-1}\right]$, for $L$, some constant, are valid in the physical time $t \varepsilon[0, L]$, which is often not long enough to make the results useful.

We will, therefore, be interested in results valid for all time for dynamical systems depending simultaneously on a fast and a slow time. It will become necessary to take a slightly special form of (1.4) and the system equations will be assumed to be almost periodic in the fast time and periodic in the slow time. The asymptotically valid method of analysis will be stated in the form of a theorem, which is a generalization of the theorem on the method of averaging in the form valid for all time [5] and the method will then be applied to a physical example which is a generalization of one discussed by Bogoliuboff and Mitropolsky in [6].

II. A theorem on a generalization of the method of averaging. In many physical examples the fast and slow behavior in (1.4) can be separated so that the system equations are of the form

$$
\dot{x}=\epsilon f(t, x)+\epsilon g(\epsilon t, x)
$$

In (2.1) $f$ and $g$ are continuous functions from $B(K)=\{(t, x): t$ is a scalar, $x$ an $n$ vector, $-\infty<t<\infty,|x| \leqq K\}$ into $R^{n}$ having continuous second partial derivatives with respect to $x$. The function $f$ is almost periodic with respect to $t$ uniformly for $|x| \leqq K$ and the function $g$ is periodic in $\epsilon t$ of period $L$, where $L$ is a fixed constant.

Our main result establishes a relationship between the almost periodic solutions of (2.1) and the corresponding periodic solution of a related averaged system in which the average is taken over $f$ alone.

That is, we define the average of $f$ to be

$$
f_{0}(\xi)=\lim _{T \rightarrow \infty} \frac{1}{T} \int_{0}^{T} f(t, \xi) d t
$$

and then consider the equation 


$$
\dot{\xi}=\epsilon f_{0}(\xi)+\epsilon g(\epsilon t, \xi) \triangleq \epsilon G(\epsilon t, \xi) .
$$

The existence and stability properties of periodic solutions of (2.3) then determine the existence and stability properties of the almost periodic solutions of (2.1) and furthermore the almost periodic solutions of (2.1) approach the periodic solutions of (2.3) as $\epsilon \rightarrow 0$. More precisely we have:

Theorem. Let $\xi^{*}(\epsilon t)$ be a periodic solution of $E q$. (2.3) of period $L / \epsilon$ in $t$ which together with a $\rho$ neighborhood remains in the interior of the set $B(K)$.

If the variational equation of $(2.3)$ with respect to $\xi^{*}$, that is,

$$
\frac{d z}{d \tau}=\frac{\partial G}{\partial \xi}\left(\tau, \xi^{*}(\tau)\right) z
$$

has no pure imaginary characteristic exponents then there exist positive constants $\epsilon_{1}$ and $\sigma, 0<\epsilon_{1} \leqq \epsilon_{0}, 0<\sigma \leqq \rho$ such that for each $\epsilon, 0<\epsilon \leqq \epsilon_{1}$, there is a unique almost periodic solution $x^{*}(t, \epsilon)$ of $(2.1)$ that satisfies $\left|x^{*}(t, \epsilon)-\xi^{*}(\epsilon t)\right|<\sigma$ for $-\infty<t<\infty$, and $x^{*}$ is continuous in $\epsilon$ and has the properties

$$
\lim _{\epsilon \rightarrow 0}\left|x^{*}(t, \epsilon)-\xi^{*}(\epsilon t)\right|=0,
$$

(ii) the stability properties, in the sense of Liapunov, are the same as those of the zero solution of (2.4).

The method of proof is to reduce system (2.1) by a series of transformations to a new system of equations and then use a corollary based on a theorem of Hale [7].

For reference we will state this theorem here but in a slightly weaker form which is a form more appropriate for our purposes.

Theorem A. Consider the system

$$
\dot{x}=A x+q(t, x, \epsilon)
$$

where $x$ is an $n$ vector, $A$ is a constant $n \times n$ matrix all of whose eigenvalues have nonzero real parts and $q$ is a continuous function from $B\left(K, \epsilon_{0}\right)=\{(t, x, \epsilon): t$ and $\epsilon$ are scalars, $x$ an n-vector, $\left.-\infty<t<\infty, 0<\epsilon \leqq \epsilon_{0},|x| \leqq K\right\}$ into $R^{n}$. The function $q$ is almost periodic in $t$ uniformly with respect to $x$ and has continuous partial derivatives with respect to $x$. Let $q_{x}$ denote the Jacobian matrix of $q$ with respect to $x$.

If

$$
\lim _{\epsilon \rightarrow 0 ; x \rightarrow 0} q_{x}(t, x, \epsilon)=0 \text { uniformly in } t
$$

and

$$
\lim _{\epsilon \rightarrow 0} q(t, 0, \epsilon)=0 \text { uniformly in } t \text {, }
$$

then there exist positive constants $\sigma$ and $\epsilon_{1}, 0<\sigma \leqq K, 0<\epsilon_{1} \leqq \epsilon_{0}$, such that for each $\epsilon, 0<\epsilon \leqq \epsilon_{1}$ there is a unique almost periodic solution $x^{*}(t, \epsilon)$ of $(2.5)$ in $B(\sigma)$ that is continuous in $\epsilon$ and $\lim _{\epsilon \rightarrow 0} x^{*}(t, \epsilon)=0$ uniformly in $t$.

Moreover, the stability properties of $x$ in the sense of Liapunov are the same as the stability properties of $y=0$ for the equation $\dot{y}=A y$.

Since when we avcrage (2.1) we are led to consider equation (2.3) which has periodic linear terms, we require the following extension of Theorem A. 
Corollary. Consider the equation

$$
\dot{x}=A(t) x+q(t, x, \epsilon)
$$

where $A(t)$ is a continuous periodic $n \times n$ matrix function of $t$, and $q$ is as in the above theorem. If none of the characteristic exponents of the solutions of

$$
\dot{y}=A(t) y
$$

are pure imaginary then there exist positive constants $\epsilon_{1}$ and $\sigma \quad 0<\epsilon_{1} \leqq \epsilon_{0}, 0<\sigma \leqq K$ and $a$ unique almost periodic solution $x^{*}(t, \epsilon)$ of (2.8) for each $\epsilon, 0<\epsilon \leqq \epsilon_{1}$, contained in $B(\sigma)$. Furthermore, $\lim _{\epsilon \rightarrow 0} x^{*}(t, \epsilon)=0$ uniformly in $t$ and $x^{*}$ is continuous in $\epsilon$.

Moreover, the stability properties of $x^{*}$, in the sense of Liapunov, are the same as the stability properties of the solution $y=0$ of (2.9).

Proof. By the Floquet representation theorem there exist a continuous periodic $n \times n$ matrix function $P(t)$ and a constant matrix $B$ such that the fundamental matrix solution of $(2.9)$ is

$$
Z(t)=P(t) \exp B t .
$$

Let $x(t)=P(t) z(t)$ and then (2.8) becomes

$$
\dot{z}=B z+P^{-1} q(t, P z, \epsilon) .
$$

The corollary follows by applying Theorem A to (2.10).

In order to use the above corollary to establish the main theorem we must change variables in (2.1). This change of variables, as in the standard form of the method of averaging, is based on the following lemma due to Bogoliuboff and Mitropolsky [1]. The form of the lemma stated here is a restricted case of the form as given by Hale [8].

Lemma. Suppose $f(t, x)$ is a continuous function from $B(\sigma)$ into $R^{n}$ and has continuous partial derivatives with respect to $x$ and is almost periodic in $t$ uniformly with respect to $x$. If the average value of $f(t, x)$ with respect to $t$ is zero, then there exists a function $w(t, x, \epsilon)$ from $B\left(\sigma, \epsilon_{0}\right)$ into $R^{n}$, almost periodic in $t$ uniformly in $x$ which has a first partial derivative with respect to $t$, derivatives of any order with respect to $x$, and another function $h(t, x, \epsilon)$ from $B\left(\sigma, \epsilon_{0}\right)$ into $R^{n}$ and such that

$$
h(t, x, \epsilon)=\frac{\partial w}{\partial t}-f(t, x)
$$

and $|h(t, x, \epsilon)|,|\partial h / \partial x(t, x, \epsilon)| \rightarrow 0$ as $\epsilon \rightarrow 0$ uniformly in $t$ and $x$ and $\epsilon w, \epsilon \partial w / \partial x \rightarrow 0$ as $\epsilon \rightarrow 0$ uniformly in $t$ and $x$.

We are now ready to prove the main theorem.

Proof. Let

$$
x=\xi+\epsilon w(t, \xi, \epsilon)
$$

where

$$
\frac{\partial w}{\partial t}-f(t, x)+f_{0}(x)=-h(t, x, \epsilon)
$$

where $w(t, x, \epsilon)$ and $h(t, x, \epsilon)$ satisfy the conditions of the above Lemma.

Substituting (2.11) in (2.1) 


$$
\begin{aligned}
\left(I+\epsilon \frac{\partial w}{\partial \xi}\right) \xi+\epsilon \frac{\partial w}{\partial t}=\epsilon f_{0}(\xi)+\epsilon\left[f(t, \xi)-f_{0}(\xi)\right]+\epsilon g(\epsilon t, \xi) & \\
& +\epsilon[f(t, \xi+\epsilon w)-f(t, \xi)]+\epsilon[g(\epsilon t, \xi+\epsilon w)-g(\epsilon t, \xi)]
\end{aligned}
$$

which after using $(2.12)$ can be written as

$$
\begin{aligned}
\xi=\epsilon\left[I+\epsilon W\left(\frac{\partial w}{\partial \xi}, \epsilon\right)\right]\left[f_{0}(\xi)+h(t, \xi, \epsilon)+g(\epsilon t, \xi)\right. & \\
& \left.+\epsilon \frac{\partial f}{\partial \xi}\left(t, \xi_{1}\right) w(t, \xi)+\epsilon \frac{\partial g}{\partial \xi}\left(\epsilon t, \xi_{2}\right) w(t, \xi)\right]
\end{aligned}
$$

where $\xi_{1}$ and $\xi_{2}$ are points on a line joining $\xi$ and $\xi+\epsilon w$ and $W$ is a matrix, $|W|$ of order zero in $\epsilon$.

Thus we have a system of the form

$$
\xi=\epsilon\left[f_{0}(\xi)+g(\epsilon t, \xi)\right]+\epsilon h(t, \xi, \epsilon)+\epsilon^{2} \Phi(\epsilon t, t, \xi, \epsilon)
$$

or

$$
\xi=\epsilon G(\epsilon t, \xi)+\epsilon h(t, \xi, \epsilon)+\epsilon^{2} \Phi(\epsilon t, t, \xi, \epsilon)
$$

where

$$
\Phi=\left(\frac{\partial f}{\partial \xi}+\frac{\partial g}{\partial \xi}\right) w+W\left(f_{0}+g+h+\epsilon \frac{\partial f}{\partial \xi}+\epsilon \frac{\partial g}{\partial \xi}\right)
$$

Now let $\xi^{*}(\epsilon t)$ be a periodic solution of period $L / \epsilon$ in $t$ of

$$
\dot{\xi}=\epsilon G(\epsilon t, \xi)
$$

and let

$$
\xi=\xi^{*}+z
$$

in (2.13). Then we have

$$
\begin{aligned}
\dot{z}=\epsilon A(\epsilon t) z+\epsilon\left[G\left(\epsilon t, \xi^{*}+z\right)-G\left(\epsilon t, \xi^{*}\right)-A(\epsilon t) z\right] \\
+\epsilon h\left(t, \xi^{*}+z, \epsilon\right)+\epsilon^{2} \Phi\left(\epsilon t, t, \xi^{*}+z, \epsilon\right)
\end{aligned}
$$

where

$$
A(\epsilon t) \triangleq \frac{\partial G}{\partial \xi}\left(\epsilon t, \xi^{*}(\epsilon t)\right)
$$

Now (2.15) is in the form to which the corollary to Theorem $A$ can be applied. Applying the corollary and tracing back through transformations (2.14) and (2.11) we have the proof of the theorem.

III. An application. A damped sinusoidally excited pendulum with a vertically oscillating support can be represented by the equation:

$$
\frac{d^{2} q}{d t^{2}}+\left(1+\frac{d^{2} h(\nu t)}{d t^{2}}\right) \sin q+F \cos \omega t=-D \frac{d q}{d t}
$$

where $q$ is the angular coordinate measured from the bottom position, $h(\nu t)$ is a continuous and periodic function of $\nu t$ with period $2 \pi, \nu=\epsilon^{-1}, 0<\epsilon \ll 1$ and where $D, F$ and $\omega$ are real positive parameters independent of $\epsilon$. 
In order to transform (3.1) into form (2.1) it is desirable to treat all but the term with $D$ in (3.1) as derivable from the Hamiltonian

$$
H(p, q, t)=\frac{1}{2}\left[p^{2}-2 p \frac{d h}{d t} \sin q-\left(\frac{d h}{d t}\right)^{2} \cos ^{2} q\right]+(1-\cos q)-q F \cos \omega t
$$

where $p$ is the momentum conjugate to the coordinate $q$ and the equations of motion are:

$\frac{d q}{d t}=p-\frac{d h}{d t} \sin q$

$\frac{d p}{d t}=-\left[-p \frac{d h}{d t} \cos q+\frac{1}{2}\left(\frac{d h}{d t}\right)^{2} \sin 2 q+\sin q-F \cos \omega t\right]-D\left(p-\frac{d h}{d t} \sin q\right)$.

We will assume $h(\nu t)=\epsilon l(\nu t)$, a quantity of order $\epsilon$, and let $\nu t=\tau$ be the fast time. Then denoting by prime derivative with respect to $\tau$ we have:

$$
\begin{aligned}
& q^{\prime}=\epsilon\left[p-l^{\prime} \sin q\right], \\
& p^{\prime}=\epsilon\left[p l^{\prime} \cos q-\frac{1}{2}\left(l^{\prime}\right)^{2} \sin 2 q-\sin q-D p+D l^{\prime} \sin q\right]+\epsilon F \cos \epsilon \omega \tau
\end{aligned}
$$

and we have the problem expressed in terms of a part periodic in the fast time (the quantity in the square bracket) and another part periodic in $\epsilon \tau$, the slow time.

The equations corresponding to (2.3) in the theorem are then

$$
\begin{aligned}
& Q^{\prime}=\epsilon P, \\
& P^{\prime}=-\epsilon\left[\frac{A^{2}}{2} \sin Q \cos Q+\sin Q+D P\right]+\epsilon F \cos \epsilon \omega \tau
\end{aligned}
$$

where $(P, Q)$ corresponds to the vector $\xi$ and where the function $l(\tau)=A \sin \tau$.

Combining the equations in (3.4) and writing the resulting equation in terms of the original time we have

$$
\frac{d^{2} Q}{d t^{2}}+D \frac{d Q}{d t}+\left(1+\frac{A^{2}}{2} \cos Q\right) \sin Q=F \cos \omega t
$$

We see from the theorem that almost periodic solutions of the original equation (3.1) approach the corresponding periodic solutions of (3.5) for $\epsilon$ sufficiently small, that is, under conditions of high frequency small amplitude support motions. If $F=0$ in (3.4) and (3.5) the well known results of Bogoliuboff and Mitropolsky are applicable. In fact, they, by a different set of transformations, obtain (3.5) with the right side zero. In this case the constant solutions $Q=0, Q=\pi, Q=\cos ^{-1}\left(2 / A^{2}\right)$ are of interest. It is seen, for example, that the constant solution $Q=\pi$, for $D>0$ is stable if $1-A^{2} / 2<0$, and thus the pendulum can execute stable motions in the neighborhood of its vertically up position. This is a result also derivable from the classical theory of the Mathieu equation.

The theorem proved here can be used to give not only results of the type given above but also other results that arise from the nonconstant periodic solutions of (3.5). Equation (3.5) is of course nonlinear and in most applications equations corresponding to (2.3) in the theorem will be nonlinear. If, however, one can assume in a given physical situation that some new parameter is small, then the well known methods of getting approximations to periodic solutions can be used. 
If in the case of (3.5), for example, $F$ can be regarded as small in some new parameter $\mu$ and if $D$ is of order zero in $\mu$, then with the aid of a simple transformation and Theorem A, it can be shown that (3.5) can have stable periodic solutions of period $2 \pi / \omega$ in the neighborhood of $Q=0$ and $Q=\pi$ and thus the pendulum can have stable almost periodic motion with frequency basis $\omega$ and $\nu r$ in the neighborhood of the bottom or top positions. If $F$ and $D$ can both be regarded as small in some parameter $\mu$ and if the value of $\omega^{2}$ is in the neighborhood of $1+A^{2} / 2$ or $A^{2} / 2-1$, one can have periodic solutions of (3.5) near $Q=0$ or $Q=\pi$ respectively and these solutions exhibit "jump" behavior similar to that associated with Duffing's Equation. One can thus predict that the pendulum can have stable almost periodic motions with jump type behavior not only in the neighborhood of the bottom position but also in the neighborhood of the top position.

The above example is typical of a large class encountered in applications. Physical systems that are nonautonomous and that have very high frequency time dependent terms have been discussed by Landau and Lifshitz [9], Bogdanoff [10] and Lowenstern [11], Sethna and Hemp [12] and others. For problems of this type the standard method of averaging is applicable. If, however, in a given system along with these high frequency time dependent terms, time dependent terms of much lower frequency occur, the results of the generalized form of the method of averaging as given here become necessary. Hemp has been able to predict, by using the method of analysis given here, the occurrence of quite remarkable physical phenomena in high order systems of the type discussed above. These results will be given in a forthcoming publication.

Acknowledgements. The author wishes to express his appreciation to Dr. J. K. Hale and Dr. K. R. Meyer for advice and help when this study was in progress. The author also wishes to thank the Air Force Office of Scientific Research for its financial support of this work under GRANT AF-AFOSR-704-66.

\section{REFERENCES}

1. N. M. Bogoliuboff and Yu. A. Mitropolsky, Asymptotic methods in the theory of nonlinear oscillations, Gordon and Breach, New York, 1962, Chaps. 5 and 6

2. J. K. Hale, Oscillations in nonlinear systems, McGraw-Hill, New York, 1963

3. Yu. A. Mitropolsky, Problems of the asymptotic theory of nonstationary vibrations, Daniel Davey, New York, 1965

4. V. M. Volosov, The method of averaging, Dokl. Acad. Nauk SSSR 137, 21-24 (1961) = Soviet Math. Dokl. 2, 221-224

5. N. M. Bogoliuboff and Yu. A. Mitropolsky, Asymptotic methods in the theory of nonlinear oscillations, Gordon and Breach, New York, 1962, p. 497

6. N. M. Bogoliuboff and Yu. A. Mitropolsky, Asymptotic methods in the theory of nonlinear oscillations, Gordon and Breach, New York, 1962, p. 404

7. J. K. Hale, Oscillations in nonlinear systems, McGraw-Hill, New York, 1963, p. 123

8. J. K. Hale, Oscillations in nonlinear systems, McGraw-Hill, New York, 1963, p. 116

9. L. D. Landau and E. M. Lifshitz, Mechanics, Pergamon Press, 1960, pp. 93-95

10. J. L. Bogdanoff, Influence on the behavior of a linear dynamical system of some imposed rapid motions of small amplitude, J. Acoust. Soc. Amer. 34, 1055-1062 (1962)

11. E. R. Lowenstern, The stabilizing effect of imposed oscillations of high frequency on a dynamical system, Philos. Mag. 13, 458 (1932)

12. P. R. Sethna and G. W. Hemp, Nonlinear oscillations of a gyroscopic pendulum with an oscillating point of suspension, Proc. Colloq. Internat. du Centre National de la Recherche Scientifique $N$ - 148, Les vibrations forcées dans les systèmes non-lineaires, 1964, pp. 375-392 